\title{
Factoring Integers with Large-Prime Variations of the Quadratic Sieve
}

\author{
Henk Boender and Herman J. J. te Riele
}

\section{CONTENTS}

1. Introduction

2. The Basic Idea

3. The Multipolynomial Quadratic Sieve

4. Efficient Calculation of The Polynomials

5. The Large-Prime Variation of MPQS

6. The Double Large-Prime Variation

7. Implementation and Experiments

Acknowledgements

References

AMS Subject Classification: 11A51, $11 Y 05$.

Keywords: factorization, multiple polynomial quadratic sieve, vector supercomputer, cluster of workstations.
This article is concerned with the large-prime variations of the multipolynomial quadratic sieve factorization method: the PMPQS (one large prime) and the PPMPQS (two). We present the results of many factorization runs with the PMPQS and PPMPQS on SGl workstations and on a Cray C90 vector computer. Experiments show that for our Cray C90 implementations PPMPQS beats PMPQS for numbers of more than 80 digits, and that this crossover point goes down with the amount of available central memory.

For PMPQS we give a formula to predict the total running time based on a short test run. The accuracy of the prediction is within $10 \%$ of the actual running time. For PPMPQS we do not have such a formula. Yet in order to provide measurements to help determining a good choice of the parameters in PPMPQS, we factored many numbers. In addition we give an experimental prediction formula for PPMPQS suitable if one wishes to factor many large numbers of about the same size.

\section{INTRODUCTION}

Let $n$ be an odd positive integer to be factored and suppose that $n$ is not a prime power. If we can find two integers $X$ and $Y$ such that

$$
X^{2} \equiv Y^{2} \bmod n
$$

then the greatest common divisor of $X-Y$ and $n$ is a nontrivial factor of $n$ if $X \not \equiv \pm Y(\bmod n)$. If $X$ and $Y$ are randomly chosen subject to (1.1), then this yields a proper factor of $n$ in at least $50 \%$ of the tries. This principle is the basis for the best known general factorization methods, namely, the multipolynomial quadratic sieve, or MPQS [Bressoud 1989; Pomerance 1985; Pomerance et al. 1988; Silverman 1987; te Riele et al. 1989], and the number field sieve, or NFS [Lenstra and Lenstra 1993]. 
In this paper we discuss and compare the single large-prime variation (PMPQS) and the double large-prime variation (PPMPQS) of MPQS. An introduction to each of these methods is given starting in Section 2. We factor many numbers ranging from 66 to 88 decimal digits, mainly with PPMPQS, on either SGI workstations or a Cray C90 vector computer.

PPMPQS is known to be faster than PMPQS "by approximately a factor of 2.5 for sufficiently large $n$ " [Lenstra and Manasse 1994], but the crossover point depends heavily on the choice of the parameters in the two methods, the computer, the available memory, and the implementation. It is stated further in [Lenstra and Manasse 1994] that PPMPQS was found to be faster than PMPQS for numbers of at least 75 decimal digits, and that the speed-up factor of 2.5 was obtained for numbers of more than 90 digits. As a comparison, a 106-digit number was factored with PMPQS in about 140 mips years, and a 107-digit number with PPMPQS in about 60 mips years, both with a factor base size of 65,500 . A 116-digit number was factored with PPMPQS in about 400 mips years, with a factor base size of 120,000 . No actual results for smaller numbers were given. Thomas Denny reports in his Master's Thesis [1993] various experiments with PPMPQS for numbers in the range of 75-95 decimal digits. From these experiments it is not clear where the crossover point for Denny's implementation lies. The largest numbers presently factored with PPMPQS are a 120-digit number done in about 825 mips years [Denny et al. 1994], and the 129-digit RSA challenge described by Martin Gardner, done in about 5000 mips years with a factor base size of 524,339 [Atkins et al. 1995].

A theoretical and practical problem with PPMPQS is the determination of the optimal parameters for a number of a given size. Since it only pays to use PPMPQS for rather large numbers, and since it is difficult to accurately predict the total running time of PPMPQS on the basis of a short test run (as contrasted with PMPQS), the precise effect of one specific choice of the parame- ters can only be measured accurately by carrying out the complete sieve part of the job. So in order to find the optimal parameter choice for a given number, that would minimize the CPU time, one would have to repeat the complete sieve job for several (10, say) different choices of the parameters. Of course, this does not make much sense since one sieve job will do to factor the number, so we decided to adopt the strategy to factor as many as possible different numbers in a not too wide decimal digits range, thus providing extensive experience with PPMPQS for many different numbers on the one hand, and contributing to a table of unfactored numbers [Brent and te Riele 1992] on the other hand. The price to pay for this strategy is that we can only give an indication of the optimal parameter choice for PPMPQS for numbers in the 65-90 decimal digits range.

We have implemented PPMPQS on an SGI workstation, and on a Cray vector computer. Some comparative experiments with PMPQS and PPMPQS on a Cray C90 indicated that for our implementation on that machine the crossover point lies around numbers having $80-85$ decimal digits. For several different choices of the parameters in PPMPQS, we have factored eight numbers in the $66-83$ digit range on an SGI workstation, and more than 70 numbers in the 67-88 digit range on a Cray C90 vector computer, as a contribution to the table in [Brent and te Riele 1992]. Most of these numbers were already tried before with the elliptic curve method (ECM), without success.

Section 2 discusses Dixon's algorithm. MPQS is described in Section 3. In Section 4 we treat the efficient generation of the polynomials in MPQS. In Section 5 the single large-prime variation of MPQS (PMPQS) is described. A known theoretical formula is worked out that helps to predict the total sieve time on the basis of a short test run. In this test run (of a few minutes CPU time, say) the speed is determined by which so-called complete and partial relations are generated during the sieve step of the algorithm; this speed is approximately constant during the whole sieve step. The 
accuracy of the prediction formula is within $10 \%$ of the actual sieve time. In Section 6 the double large-prime variation of MPQS is described, and an experimental prediction formula is given that has a restricted scope in the sense that it only applies to numbers of roughly the same size, and for a fixed choice of the parameters of the algorithm. In addition, for one particular number of 80 decimal digits, we have determined the optimal choice of one (of the four) parameters in PPMPQS as an illustration of the fact that this optimum is attained for a rather wide range of this parameter. Section 7 covers implementation aspects and discusses our experiments, including a comparison of our PMPQS- and PPMPQS-implementations for 71-, 87-, and 99-digit numbers. The paper closes with data from 81 factorizations.

\section{THE BASIC IDEA}

The algorithm described now is due to Dixon, who based it on the continued fraction method of Morrison and Brillhart [1975]. It is not efficient in practice compared to almost any other method, but it shows clearly the idea behind finding $X$ and $Y$. So we mention it mainly for didactical reasons.

For $x \in \mathbb{Z}$ such that $|x|>\sqrt{n}$, define

$$
g(x): \equiv x^{2} \bmod n .
$$

(The notation $\sqrt{n}$ means the positive square root of $n$.) Suppose that we have a finite subset $J \subset \mathbb{Z}$ such that $\prod_{x \in J} g(x)$ is a square. Then we can take

$$
X=\sqrt{\prod_{x \in J} g(x)}, \quad Y=\prod_{x \in J} x .
$$

A problem is how to determine $J$.

Choose a positive integer $B_{1}$, let $\pi=\pi\left(B_{1}\right)$ be the number of primes $\leq B_{1}$, and let $\left\{p_{1}, p_{2}, \ldots, p_{\pi}\right\}$ be the set of such primes. Suppose that we have a set $T$ of $t>\pi$ numbers $g(x)$ only composed of primes $\leq B_{1}$, that is,

$$
g(x)=p_{1}^{e_{1}(x)} p_{2}^{e_{2}(x)} \ldots p_{\pi}^{e_{\pi}(x)},
$$

where $e_{i}(x)$ is the exponent of $p_{i}$ in $g(x)$. Then

$$
\prod_{x \in J} g(x)=\prod_{i=1}^{\pi} p_{i}^{\sum_{x \in J} e_{i}(x)}
$$

This is a square if and only if

$$
\sum_{x \in J} e_{i}(x) \equiv 0 \bmod 2, \quad \text { for } i=1,2, \ldots, \pi .
$$

Since $|T|=t>\pi$, there exists a nontrivial solution of this linear system of equations over GF(2). A solution can be found using Gaussian elimination. This yields at least $t-\pi$ useful subsets $J$.

\section{THE MULTIPOLYNOMIAL QUADRATIC SIEVE}

Dixon's algorithm does not tell us how to find $T$ efficiently. Building on previous work of Kraitchik [1929], Lehmer and Powers [1931], Morrison and Brillhart [1975], and Schroeppel, Carl Pomerance [1982] introduced the quadratic sieve algorithm. It works with the quadratic polynomial

$$
g(x)=(x+\lfloor\sqrt{n}\rfloor)^{2}-n,
$$

where $x$ runs over the integers in $\left(-n^{\varepsilon}, n^{\varepsilon}\right)$, so that $g(x)=O\left(n^{1 / 2+\varepsilon}\right)$. With this $g(x)$ the set $T$ may be built up, where some of the numbers $g(x)$ can be factored completely by a cheap sieve process because $g(x)$ is a polynomial (this is much more efficient than trial division or any other factoring method). We could also use a sieve process in Dixon's algorithm if we choose random numbers $x$ in an arithmetic progression like $x, x+1, x+2, \ldots$. However, in practice this single polynomial $g(x)$ (or an arithmetic progression in Dixon's algorithm) does not give rise to a sufficiently large set $T$ (with $t>\pi$ elements) in a reasonable amount of time. The reason for this is that the interval $\left(-n^{\varepsilon}, n^{\varepsilon}\right)$ is large when $n$ is large and, since $g(x)=O\left(n^{1 / 2+\varepsilon}\right)$ (which is large), most numbers $g(x)$ are not likely to factor over a set of small primes. P. L. Montgomery found an efficient way to use several polynomials (thus introducing a simple way to run the algorithm in parallel), so that the numbers $x$ can be taken from much smaller intervals rather than 
from one single very large interval. The average polynomial values then are smaller than the average value of $g$ and are thus more likely to factor over small primes than the $g(x)$-values. If all the numbers in a small interval have been considered, we can pass to a next polynomial and try again. We describe here the resulting multipolynomial quadratic sieve method. We remark that Davis and Holdridge [1983] already had a multipolynomial version before Montgomery came up with his new idea. In fact, Montgomery's method is based on that of Davis and Holdridge, but it is slightly more efficient.

Suppose that we have integer numbers $x, U(x)$, $V(x)$, and $W(x)$ such that

$$
U^{2}(x) \equiv V^{2}(x) W(x) \bmod n \quad \text { for all } x \in \mathbb{Z} \text {. }
$$

If $J \subset \mathbb{Z}$ is a finite subset such that $\prod_{x \in J} W(x)$ is a square, we can take

$$
X=\prod_{x \in J} V(x) \sqrt{\prod_{x \in J} W(x)}, \quad Y=\prod_{x \in J} U(x) .
$$

In practice we choose $U(x)=a^{2} x+b, V(x)=a$, and $W(x)=a^{2} x^{2}+2 b x+c$, with $|x| \leq M$ (where $M$ is a parameter we choose beforehand) and where $a, b$ and $c$ are integers that satisfy the following conditions [Bressoud 1989, p. 117]:

$$
\begin{aligned}
a^{2} & \approx \sqrt{2 n} / M, \\
b^{2}-n & =a^{2} c, \\
|b| & <a^{2} / 2 .
\end{aligned}
$$

In the next section we describe how $a, b$ and $c$ are to be calculated.

$W(x)$ plays the role of $g(x)$ in Dixon's algorithm. In order to determine the subset $J$, we choose an upper bound $B_{1}$ for the primes. We want to have many $W(x)$-values that consist of primes $\leq B_{1}$. However, only roughly half of the primes below $B_{1}$ can occur as a prime divisor of $W(x)$. Namely, if a prime $p$ divides $W(x)$, then $p \mid a^{2} W(x)$ and thus $p \mid\left(a^{2} x+b\right)^{2}-n$, which means that $n$ is a quadratic residue modulo $p$. This leads to the definition of the factor base $\mathcal{F}$ :

$$
\mathcal{F}=\left\{\text { prime powers } q=p^{k} \leq B_{1}:\left(\frac{n}{p}\right)=1\right\} .
$$

(Of course, a prime can divide $W(x)$ more than once, so we also have to account for prime powers.) Note that $\mathcal{F}$ is independent of the choices of $a, b$ and $c$, so we can use the same factor base for every proper choice of $a, b$ and $c$.

Since $W(x)$ is more likely to be divisible by small primes than by large primes, it is advantageous that the factor base contains many small primes. We can construct such a factor base by multiplying the number $n$ to be factored by a suitable small integer $m$, called the multiplier, and factoring $m n$ rather than $n$ [Pomerance et al. 1988, p. 391].

For a given $q \in \mathcal{F}$ the values of $x$ for which $q$ divides $W(x)$ can be found as follows. Compute the solution $t=t_{q}$ of the congruence equation

$$
t^{2} \equiv n \bmod q, \quad \text { for } 0<t \leq q / 2
$$

[Riesel 1985, pp. 212 and 287-288]. This has to be done only once during the algorithm. Now, if $q \mid W\left(x_{0}\right)$, then $q \mid\left(a^{2} x_{0}+b\right)^{2}-n$ and thus

$$
x_{0} \equiv a^{-2}\left( \pm t_{q}-b\right) \bmod q
$$

provided that $\operatorname{gcd}(a, q)=1$. This is guaranteed by the choice of $a$ (see Section 4). For each proper choice of $a$ we compute $a^{-2} \bmod q$ for all $q \in \mathcal{F}$. In the next section we describe how these computations can be done. Furthermore, since $W(x)$ is a quadratic polynomial, $q$ divides $W\left(x_{0}+l q\right)$, for $l \in \mathbb{Z}$. So we can calculate efficiently the places where an element of $\mathcal{F}$ divides the $W$-values. This idea originated from Schroeppel.

Define the report threshold $\mathrm{RT}$ as the average of $\log |W(x)|$ on the interval $[-M, M]$, which is approximately $\log \left(\frac{1}{2} M \sqrt{n / 2}\right)$. Initialize a sieve array $\mathrm{SI}(-M, M)$ to zero and sieve with each $q=p^{k} \in \mathcal{F}$, i.e., add $\log p$ to $\operatorname{SI}\left(x_{0}+l q\right)$ for all $l \in \mathbb{Z}$ such that $x_{0}+l q$ is in the interval $[-M, M]$. For those numbers $x$ for which $\mathrm{SI}(x) \geq \mathrm{RT}, W(x)$ is a good candidate for fully factoring over the factor base. In 
general, the time spent on sieving takes more than $85 \%$ of the total computing time.

Since sieving with small primes is expensive, it is customary not to sieve with the primes and prime powers $\leq \mathrm{QT}$, where QT is some suitably chosen threshold value. In order not to lose $W(x)$-values divisible by such small primes, the report threshold $\mathrm{RT}$ will be lowered by the amount $\sum_{p^{k} \leq \mathrm{QT}} \log p$. After the sieve step and the selection of those $x$ for which $\mathrm{SI}(x) \geq \mathrm{RT}$, the prime factors of the corresponding $W(x)$ are found by comparison, for all $q \in \mathcal{F}$, of $x$ with the two values of $x_{0}$ in (3.5) (which are computed and stored after the factor base has been computed). In this way, $W(x)$-values divisible by one or more of the small primes omitted during the sieving phase are not lost. If QT is suitably chosen, this can save a considerable amount of sieve time. This refinement of MPQS is known as the small-prime variation.

\section{EFFICIENT CALCULATION OF THE POLYNOMIALS}

Choose integers $r$ and $k$ such that $1<k<r$ (typical choices are $r=30$ and $k=3$, for example). Generate primes $g_{1}, g_{2}, \ldots, g_{r}$, the so-called $g$-primes, such that (i) $g_{i} \approx(\sqrt{2 n} / M)^{1 /(2 k)}$, (ii) $\left(\frac{n}{g_{i}}\right)=1$, and (iii) $\operatorname{gcd}\left(g_{i}, q\right)=1$, for $i=1,2, \ldots, r$ and for all $q \in \mathcal{F}$. Let

$$
a=g_{i_{1}} g_{i_{2}} \ldots g_{i_{k}},
$$

be the product of $k g$-primes, with $1 \leq i_{1}<i_{2}<$ $\cdots<i_{k} \leq r$. Because of (i), this $a$ satisfies condition (3.2).

Let $b_{i}$ be a solution of the congruence equation

$$
t^{2} \equiv n \bmod g_{i}^{2},
$$

where $i=1,2, \ldots, r$. Solve the system of congruence equations (for a specific choice of the signs)

$$
\begin{aligned}
x & \equiv b_{i_{1}} \bmod g_{i_{1}}^{2}, \\
x & \equiv \pm b_{i_{2}} \bmod g_{i_{2}}^{2}, \\
& \vdots \\
x & \equiv \pm b_{i_{k}} \bmod g_{i_{k}}^{2},
\end{aligned}
$$

by means of the Chinese Remainder Theorem. Let $b$ be the solution of this system of equations. Then $b^{2} \equiv n \bmod a^{2}$, so that condition (3.3) holds with

$$
c=\left(b^{2}-n\right) / a^{2} .
$$

If $b \geq a^{2} / 2$, we replace $b$ by $b-a^{2}$ in order to satisfy condition (3.4). Since there are $2^{k-1}$ possible combinations of signs in (4.1), the number of polynomials that can be calculated with one set of $r$ $g$-primes and a fixed $k$ is $2^{k-1}\left(\begin{array}{l}r \\ k\end{array}\right)$.

If a new $a$ has to be chosen, new sieve numbers $x_{0}$ subject to (3.5) must be computed. Since $a=$ $g_{i_{1}} g_{i_{2}} \ldots g_{i_{k}}$, we can use

$$
a^{-2} \bmod q=g_{i_{1}}^{-2} g_{i_{2}}^{-2} \ldots g_{i_{k}}^{-2} \bmod q .
$$

Therefore, with the generation of the $g$-primes we also compute and store the numbers $g_{i}^{-2} \bmod q$, where $i=1,2, \ldots, r$, for all the prime powers $q$ in the factor base.

For a fixed $a$, Alford and Pomerance [1995] developed a method to compute iteratively all the other values of $b$ (and thus $c$ ) from a given initial value of $b$ (see also [Peralta $\geq 1996]$ ). They also pointed out how the two solutions in the interval $[0, q)$ of the congruence equation $W(x) \equiv$ $0 \bmod q$ can be calculated from the zeros $\bmod q$ of a "previous" polynomial. With this improvement we obtain the self-initializing variation of MPQS. It has the advantage that it can change polynomials cheaply, so a shorter sieve interval can be used.

We have implemented this variation on an SGI workstation and on a Cray C90 vector computer. Some speed-up was observed on an SGI workstation when we reduced the length of the sieve interval, but other effects like an increasing loop overhead in the sieving step interfere with this in the opposite direction.

On a vector computer such as the Cray C90, reducing the length of the sieve interval reduces the vector lengths in the sieving step and, consequently, the efficiency of the vectorization. Therefore, we decided not to use the self-initializing variation of the quadratic sieve in our experiments. 


\section{THE LARGE-PRIME VARIATION OF MPQS}

The large-prime variation of MPQS incorporates the following improvement, which is based on a step in the continued fraction algorithm of Morrison and Brillhart [1975]. $W(x)$ is allowed to have a factor $R>B_{1}$ that is not composed of primes from the factor base. If the cofactor $R$ (after dividing out all factor base primes in $W(x))$ is less than or equal to $B_{1}^{2}$, it must be a prime. In order to restrict the amount of disk space needed for storage of the relations (3.1), we only accept factors $R \leq B_{2}$, where $B_{2}$ is a parameter we choose beforehand. In practice we choose $B_{2}$ in such a way that $B_{2} / B_{1}$ is a number between 10 and 100 . We have to lower the report threshold by $\log \left(B_{2}\right)$ in order to find these $W(x)$-values after sieving.

If we have found two $W(x)$-values with the same $R$, multiplication of the corresponding relations (3.1) yields a relation of the form (3.1), where $W(x)$ only consists of prime powers $q \in \mathcal{F}$ (and $R$ is moved to $V(x)$ ).

A relation of the form (3.1), where $W(x)$ only consists of primes $q \in \mathcal{F}$, is called a complete relation. If $W(x)$ has one prime factor $R \leq B_{2}$ (and the others are in $\mathcal{F})$, then the relation is called a partial relation.

We wish to compute $E$, the expected number of complete relations coming from a given number of $r$ partial relations. Let

$$
Q=\left\{\text { primes } q: B_{1}<q \leq B_{2},\left(\frac{n}{q}\right)=1\right\} .
$$

The elements of $Q$ are called large primes. Let $P_{q}$ be the probability that a large prime $q$ occurs in a partial relation. Lenstra and Manasse [1994] assume that

$$
P_{q} \approx q^{-\alpha} / \sum_{p \in Q} p^{-\alpha}
$$

for some positive constant $\alpha<1$ that should be determined experimentally. They report that $\alpha \in$ $\left[\frac{2}{3}, \frac{3}{4}\right]$ gives a reasonable fit with their experimental results. Denny [1993, pp. 44-49] takes $\alpha=0.775$.
From [Lenstra and Manasse 1994] it follows that

$$
E=r-\# Q+\sum_{q \in \mathcal{Q}}\left(1-P_{q}\right)^{r} .
$$

We apply the binomial formula of Newton and use approximation (5.1) to find

$$
E \approx \sum_{i=2}^{r}(-1)^{i}\left(\begin{array}{l}
r \\
i
\end{array}\right)\left(\sum_{q \in \mathcal{Q}} q^{-\alpha}\right)^{-i} \sum_{q \in \mathcal{Q}} q^{-\alpha i} .
$$

Since $\pi(t) \sim t / \log t$ as $t \rightarrow \infty$, we have

$$
\sum_{p \leq x} p^{-u} \approx \int_{2}^{x} t^{-u} d(t / \log t)
$$

with $p$ prime, $x \in \mathbb{R}_{\geq 2}, u \in \mathbb{R}_{>0}$. Hence for $u>0$ we have

$$
\sum_{q \in \mathcal{Q}} q^{-u} \approx \frac{1}{2} \int_{B_{1}}^{B_{2}} t^{-u} d(t / \log t) .
$$

To compute the last integral we first use partial integration and then substitute $s=(1-u) \log t$. We get

$$
\begin{aligned}
& \int_{B_{1}}^{B_{2}} t^{-u} d(t / \log t)=B_{2}^{1-u} / \log B_{2}-B_{1}^{1-u} / \log B_{1} \\
& +u\left\{\operatorname{Ei}\left((1-u) \log B_{2}\right)-\operatorname{Ei}\left((1-u) \log B_{1}\right)\right\},
\end{aligned}
$$

where $\operatorname{Ei}(x)=\int_{-\infty}^{x}\left(e^{s} / s\right) d s$ is the exponential integral. Now combine the last three displayed equations for the appropriate choices of $u$ to get an approximation for $E$. In approximation (5.2) we sum from $i=2$ to $i=5$ and forget about the higherorder terms to get a formula for an approximation of $E$ that we can use in practice (given $B_{1}, B_{2}, r$, and $\alpha$ ).

The experiments summarized in Table 1 show that our approximation works well if $\alpha=0.73$. The table shows, for each example run, the number $r$ of partial relations, the actual number of complete relations derived from these partial relations, and the estimated number of complete relations. An approximation of $E$ can be used to predict the computing time. 


\begin{tabular}{|cccrrr|}
\hline$n$ & $B_{1} / 10^{4}$ & $B_{2} / B_{1}$ & \multicolumn{1}{c}{$r$} & actual & estim. \\
\hline C75 & 30 & 20.0 & 37472 & 4790 & 4966 \\
C80 & 10 & 60.0 & 15918 & 1121 & 1209 \\
C80 & 30 & 167 & 68195 & 4113 & 4150 \\
C84 & 80 & 25.0 & 96138 & 10894 & 11148 \\
C88 & 50 & 100 & 94651 & 6605 & 6736 \\
C88 & 75 & 100 & 148403 & 11455 & 11211 \\
C88 & 75 & 100 & 158214 & 12830 & 12657 \\
C88 & 75 & 100 & 146983 & 11051 & 11008 \\
C88 & 75 & 100 & 150327 & 11498 & 11488 \\
C88 & 70 & 100 & 148016 & 12116 & 11827 \\
\hline
\end{tabular}

TABLE 1. For ten composite numbers and bounds $B_{1}, B_{2}$, we list the number $r$ of partial relations, and the actual and estimated number of complete relations (last two columns). As usual, $\mathrm{C} x$ denotes a composite number with $x$ decimal digits.

To determine the best value of $\alpha$, we wrote a program in Maple that, given $\alpha$, computes the absolute value of the difference of the actual number of complete relations and the estimated number of complete relations for each of fifteen test numbers. Then we summed the fifteen absolute values of the differences, thus obtaining for each $\alpha$ a sum of absolute values. It turned out that $\alpha=0.73$ gave rise to the smallest sum.

\section{THE DOUBLE LARGE-PRIME VARIATION}

In the large-prime variation of MPQS we allow $W(x)$ in (3.1) to have a prime factor $R$ with $B_{1}<$ $R \leq B_{2}$. In the double large-prime variation of MPQS we also let $W(x)$ have a factor $R \leq B_{2}^{2}$ composed of two primes $>B_{1}$. In this case we call such a relation a partial-partial relation (pp-relation for short). Now the problem of finding combinations of partial and partial-partial relations that yield a complete relation can be formulated as finding cycles in an undirected graph: the vertices are the large primes and two vertices (primes) are connected by an edge if there is a pp-relation in which both primes occur. A partial relation is represented by adding 1 as a vertex to the graph. We consider this partial relation as a pp-relation where one of the large primes is 1 . So an edge in the graph corresponds to a partial or partial-partial relation and a cycle corresponds to a set of relations with the following property: if we multiply these relations, then all the large primes in the product occur to an even power. Hence, for the linear algebra step this set can be viewed as a complete relation. To avoid dependent relations one only has to find the basic cycles of the graph.

The number of complete relations coming from the pp-relations is much more difficult to predict than that coming from the partial relations. One has to know how the number of basic cycles in a graph with given vertices varies when edges are added more or less randomly. Having a basic cycle is a monotone increasing property [Bollobás 1985, p. 33] that can appear rather suddenly [Erdős and Rényi 1959; 1960; 1961]. An algorithm for finding the basic cycles in a graph can be found in [Paton 1969].

If $R$ is prime then we require $R<B_{2}$ in order to restrict the total number of relations (in our experience partial relations with $B_{2} \leq R<B_{1}^{2}$ do not contribute much to the total number of complete relations). If $R$ is composite, its large prime factors can be found, e.g., by using Shanks' SQUFOF algorithm [Riesel 1985, pp. 191-199]. This algorithm has the advantage that most numbers that occur during its execution are in absolute value not larger than $2 \sqrt{R}$.

We want to estimate the time that PPMPQS spends on the sieve step for numbers $n$ of about $d$ decimal digits, given $B_{1}, M, B_{2}$, and QT. To that end, let

$$
\begin{aligned}
n_{f} & =\text { number of elements in the factor base } \\
n_{c} & =\text { number of complete relations, } \\
f_{1} & =n_{c} / n_{f} \\
n_{1} & =\text { number of partial relations, } \\
n_{2} & =\text { number of pp-relations, } \\
f_{2} & =n_{2} / n_{1} \\
T_{s} & =\text { sieve time. }
\end{aligned}
$$

During the sieve step, the numbers $n_{c}, n_{1}$ and $n_{2}$ grow (more or less) linearly with the time, so that 


\begin{tabular}{|c|c|c|c|c|c|c|c|c|c|c|c|c|c|c|c|c|}
\hline$\#$ & 33 & 35 & 37 & 44 & 42 & 38 & 48 & 40 & 47 & 34 & 39 & 36 & 46 & 41 & 32 & 43 \\
\hline$m$ & 109 & 37 & 1 & 109 & 1 & 109 & 5 & 29 & 1 & 1 & 1 & 7 & 43 & 1 & 1 & 41 \\
\hline$f_{1}$ & 0.243 & 0.244 & 0.255 & 0.269 & 0.275 & 0.297 & 0.301 & 0.310 & 0.320 & 0.325 & 0.331 & 0.346 & 0.348 & 0.349 & 0.352 & 0.363 \\
\hline$f_{2}$ & 5.98 & 5.79 & 4.04 & 3.68 & 2.75 & 2.37 & 2.13 & 2.29 & 1.70 & 1.64 & 1.14 & 0.906 & 0.961 & 0.862 & 0.760 & 0.798 \\
\hline
\end{tabular}

TABLE 2. Values of $f_{1}$ and $f_{2}$ measured for 16 numbers $n$ from Table 7 (identified by the number in the first row). We used $d=86, B_{1}=5 \times 10^{5}, M=1.5 \times 10^{6}, B_{2} / B_{1}=20$, and QT $=40$, with multiplier $m$.

also the fraction $f_{1}$ grows linearly, and $f_{2}$ stays more or less constant (after the sieve step has been running for a short time). We observed that the values of the fractions $f_{1}$ and $f_{2}$, measured after completion of the sieve step, seem to be connected; see Table 2.

The table suggests that $f_{2}$ is an exponential function of $f_{1}$, that is,

$$
f_{2}=a e^{b f_{1}}
$$

for some constants $a$ and $b$. Based on the table, we estimated $a=315$ and $b=-16.5$. Since $\log f_{2}=$ $\log a+b f_{1}$, it follows that

$$
n_{c}=\frac{1}{b}\left(\log f_{2}-\log a\right) \cdot n_{f}
$$

If $u$ is the time needed to generate one complete relation, we obtain the following approximation for the sieve time $T_{s}$ :

$$
T_{s} \approx\left(0.349-0.061 \log f_{2}\right) \cdot u \cdot n_{f}
$$

We can estimate $u$ and $f_{2}$ by letting the program run for a short while, five minutes say. The measurements shown in Table 3, pertaining to runs on

\begin{tabular}{|rrccccc|}
\hline$\#$ & $m$ & $u$ & $f_{2}$ & $n_{f}$ & $T_{s}$ & approx. \\
\hline 21 & 19 & $5.140 \mathrm{~s}$ & 1.1945 & 20741 & $9.8 \mathrm{~h}$ & $10.0 \mathrm{~h}$ \\
22 & 1 & $4.518 \mathrm{~s}$ & 0.7646 & 20744 & $9.8 \mathrm{~h}$ & $9.50 \mathrm{~h}$ \\
24 & 1 & $3.357 \mathrm{~s}$ & 1.4378 & 20930 & $6.0 \mathrm{~h}$ & $6.37 \mathrm{~h}$ \\
31 & 1 & $4.226 \mathrm{~s}$ & 1.0866 & 24641 & $10.0 \mathrm{~h}$ & $9.94 \mathrm{~h}$ \\
48 & 5 & $8.785 \mathrm{~s}$ & 2.1364 & 20911 & $15.4 \mathrm{~h}$ & $15.4 \mathrm{~h}$ \\
\hline
\end{tabular}

TABLE 3. Tests of approximation (6.1). For five composite numbers from Table 7 (identified by the number in the first column), we measured the actual value of $T_{s}$ and computed the value predicted by the approximation (last column). the Cray C90 of several 85- and 86-digit numbers, suggest that the estimate works well.

Consequently, approximation (6.1) can be used to obtain a good estimate of $T_{s}$ in the PPMPQS algorithm for numbers of about the same size, and fixed parameters $B_{1}, M, B_{2}$, and QT. For numbers in another range, or if we wish to change the parameters, some experiments have to be done to determine the total sieve time under these new conditions, by which the coefficients in (6.1) can be estimated.

In order to test the dependency of $T_{s}$ on $B_{2}$, we carried out on the Cray C90 the complete sieve step of PPMPQS for the 80-digit number

$$
\frac{75^{64}+1}{2 \cdot 224914177 \cdot 151113908786421917036806943723393},
$$

which has the two prime factors

68799038786512319388821350925569 and 215768091527974049646247615957101365677594246657.

We kept $B_{1}=10^{5}, M=3 \times 10^{6}$, and QT $=50$ fixed, and tried various values of $B_{2}$. The statistics are shown in Table 4.

In the partial relations we allowed the large prime $R$ to be less than $B_{1}^{2}$. (We get these relations free, because $R<B_{1}^{2}$ implies that $R$ is prime.) For $B_{1}=10^{5}$ the number of elements in the factor base is 4806 . The sieving was continued until the total number of complete relations, including those generated by the partial relations and the partialpartial relations, surpassed this number. We only measure the total number of complete relations obtained so far at selected points in our program, so the actual total number of complete relations is 


\begin{tabular}{|rcrrrrrr|}
\hline$B_{2} / B_{1}$ & $T_{s}$ & \multicolumn{1}{c}{$n_{c}$} & \multicolumn{1}{c}{$n_{1}$} & $n_{c, 1}$ & \multicolumn{1}{c|}{$n_{2}$} & $n_{c, 2}$ & total \\
\hline 30 & $8.64 \mathrm{~h}$ & 1036 & 129318 & 1661 & 29143 & 2121 & 4818 \\
60 & $7.06 \mathrm{~h}$ & 871 & 117532 & 1249 & 51929 & 2739 & 4859 \\
100 & $6.49 \mathrm{~h}$ & 775 & 109506 & 1025 & 76324 & 3070 & 4870 \\
200 & $6.02 \mathrm{~h}$ & 685 & 99474 & 795 & 123001 & 3339 & 4819 \\
400 & $5.67 \mathrm{~h}$ & 618 & 91332 & 634 & 193278 & 3598 & 4850 \\
600 & $5.71 \mathrm{~h}$ & 578 & 87265 & 568 & 243015 & 3698 & 4844 \\
800 & $5.62 \mathrm{~h}$ & 563 & 84926 & 531 & 291177 & 3766 & 4869 \\
1000 & $5.75 \mathrm{~h}$ & 546 & 83082 & 501 & 333726 & 3796 & 4843 \\
1600 & $6.19 \mathrm{~h}$ & 521 & 79960 & 464 & 445526 & 3860 & 4845 \\
\hline
\end{tabular}

TABLE 4. Number of relations as a function of $B_{2}$, for the factorization of $(6.2)$ with $B_{1}=10^{5}$, $M=3 \times 10^{6}$, and $\mathrm{QT}=50$. The column $n_{c, 1}$ is the number of complete relations generated by the $n_{1}$ partial relations, and $n_{c, 2}$ is the number of complete relations generated by combining the partial relations (with different large primes) and the $n_{2}$ pp-relations. "Total" is the sum $n_{c}+n_{c, 1}+n_{c, 2}$.

usually somewhat larger than the number of elements in the factor base.

As we increase $B_{2} / B_{1}$, the program generates more partial-partial relations and less complete and less partial relations in a given amount of sieve time. For $30 \leq B_{2} / B_{1} \leq 400$, the gain in complete relations $\left(n_{c, 2}\right)$ generated by the pp-relations $\left(n_{2}\right)$ more than sufficiently compensates for the loss of complete relations directly found by the sieve $\left(n_{c}\right)$ and the loss of complete relations $\left(n_{c, 1}\right)$ generated by the partial relations $\left(n_{1}\right)$. As a result, the total sieve time $T_{s}$ goes down. For $B_{2} / B_{1}>1000$, however, the increase in size of the large primes in the partial and partial-partial relations is responsible for a decrease in the number of complete relations derived from these relations, and also the time that SQUFOF needs to find the two large primes in a pp-relation increases, so now the resulting total sieve time increases. Consequently, the minimal sieve time is reached if we choose $B_{2} / B_{1}$ in the interval $400<B_{2} / B_{1}<1000$. In that interval the total sieve time is only slightly varying. We conclude that, in order also to minimize the amount of memory for storage of the relations, the optimal choice of $B_{2} / B_{1}$ is about 400 .

\section{IMPLEMENTATION AND EXPERIMENTS}

For our PMPQS-experiments we used the implementation described in [te Riele et al. 1989]. Almost all our subroutines are written in Fortran.

We originally implemented the PPMPQS algorithm on a supercomputer like the Cray C90 vector computer. We used the same implementation on Silicon Graphics workstations. (We now have written a program especially designed for workstations).

The sieve operations (i.e., additions of $\log p$ to an element of the sieve array) are done in 64-bits floating-point arithmetic on Cray and in 32-bits on SGI. The maximum speed we obtained (in millions of sieve operations per second) was 3.3 on the Silicon Graphics, 110 on the Cray Y-MP [te Riele et al. 1991] and 270 on the Cray C90. The maximum speed was 5.7 when we used the workstation version of our program.

We used a package of Winter in order to carry out multiprecision integer arithmetic.

The large prime $R$ occurring in the partial relations was accepted if $B_{1}<R<B_{2}$ and rejected if $B_{2} \leq R<B_{1}^{2}$.

We have implemented Paton's cycle-finding algorithm [1969] and used it as a preprocessing step for the Gaussian elimination step in PPMPQS.

An algorithm for just counting (not finding) the basic cycles [Lenstra and Manasse 1994, pp. 789790; Denny 1993, pp. 61-64] was implemented by us as a tool to check during the sieve part of PPMPQS whether sufficiently many relations (complete, partial, and partial-partial) were collected.

The method used to do the Gaussian elimination modulo 2 is described in [Parkinson and Wunderlich 1984]. The elements of the bit-array are packed in words of 64 bits (on the Cray computers) or 32 bits (on the Silicon Graphics). This allows the use of the exclusive-or operation with the column vectors of the array, which is very efficient. The total Gaussian elimination step (including finding basic cycles) accounts for less than $0.6 \%$ of the total work of the PPMPQS algorithm. 


\begin{tabular}{|c|c|c|c|c|c|c|c|c|c|c|c|c|c|c|}
\hline & \multirow[b]{2}{*}{$B_{1}$} & \multirow[b]{2}{*}{$n_{f}$} & \multirow[b]{2}{*}{$B_{2} / B_{1}$} & \multirow[b]{2}{*}{$M$} & \multicolumn{4}{|c|}{ PMPQS } & \multicolumn{6}{|c|}{ PPMPQS } \\
\hline & & & & & $T_{\boldsymbol{s}}$ & $n_{c}$ & $n_{1}$ & $n_{c, 1}$ & $T_{s}$ & $n_{c}$ & $n_{1}$ & $n_{c, 1}$ & $n_{2}$ & $n_{c, 2}$ \\
\hline C71 & $3 \times 10^{5}$ & 12979 & 20 & $5.0 \times 10^{5}$ & $0.58 \mathrm{~h}$ & 10204 & 17993 & 2784 & $0.55 \mathrm{~h}$ & 5063 & 36468 & 4709 & 42617 & 3400 \\
\hline $\mathrm{C} 71$ & $6 \times 10^{5}$ & 24510 & 20 & $5.0 \times 10^{5}$ & $0.56 \mathrm{~h}$ & 20827 & 23794 & 3703 & $0.96 \mathrm{~h}$ & 10868 & 68019 & 8383 & 70395 & 5389 \\
\hline C71 & $6 \times 10^{5}$ & 24510 & 40 & $5.0 \times 10^{5}$ & $0.55 \mathrm{~h}$ & 20312 & 30399 & 4209 & $1.28 \mathrm{~h}$ & 9817 & 80017 & 7390 & 132290 & 7412 \\
\hline $\mathrm{C} 71$ & $6 \times 10^{5}$ & 24510 & 40 & $2.5 \times 10^{6}$ & $0.29 \mathrm{~h}$ & 20196 & 31034 & 4359 & $1.21 \mathrm{~h}$ & 9803 & 81612 & 7499 & 138147 & 7969 \\
\hline $\mathrm{C} 80$ & $10^{5}$ & 4806 & 400 & $3.0 \times 10^{6}$ & $13.4 \mathrm{~h}$ & 1580 & 49143 & 3229 & $5.67 \mathrm{~h}$ & 618 & 91332 & 634 & 193278 & 3598 \\
\hline $\mathrm{C} 87$ & $5 \times 10^{5}$ & 20838 & 20 & $2.5 \times 10^{6}$ & $16.4 \mathrm{~h}$ & 9902 & 70029 & 10940 & $11.9 \mathrm{~h}$ & 7009 & 63089 & 8220 & 57513 & 5620 \\
\hline
\end{tabular}

TABLE 5. Comparison of PMPQS and PPMPQS. The C71 and C87 are listed on this page, the C80 in (6.2) on page 264 .

In order to compare PMPQS with PPMPQS we have run our implementations of these algorithms on the Cray C90 for the 71-digit number

$$
\mathrm{C} 71=\left(10^{71}-1\right) / 9
$$

and for the 87-digit cofactor

$\mathrm{C} 87=1360245925758378639396610479463908049304-$ 23542841197990430220444148923901462079070640121

of $72^{99}+1$. For $\mathrm{C} 71$, four experiments with different combinations of $B_{1}, B_{2} / B_{1}$, and $M$ were carried out where in the second, third and fourth experiment only one of the three parameters was changed compared with the previous experiment. The value of $\mathrm{QT}$ was kept fixed at 40 . For $\mathrm{C} 80$ from (6.2), which was treated in the previous section with PPMPQS, we made a comparison run with $\mathrm{PMPQS}$ for $B_{1}=10^{5}, M=3 \times 10^{6}, \mathrm{QT}=50$, and $B_{2} / B_{1}=400$ (the optimal choice for PPMPQS). The results are given in Table 5 .

For C71, the parameter choice $B_{1}=3 \times 10^{5}$, $B_{2} / B_{1}=20$, and $M=5 \times 10^{5}$ yields a somewhat smaller sieve time for PPMPQS (0.55 CPU hours) than for PMPQS (0.58), but if we allow more memory use by choosing $B_{1}=6 \times 10^{5}$ and $M=2.5 \times 10^{6}$ (and $B_{2} / B_{1}=40$ ), then PMPQS beats PPMPQS ( 0.29 vs. 1.21$)$. Increasing the length of the sieve interval ( $M$ from $5 \times 10^{5}$ to $2.5 \times 10^{6}$ ) particularly improves the efficiency of PMPQS (and, to a lesser extent, of PPMPQS). For C87, with the parameter choice $B_{1}=5 \times 10^{5}, B_{2} / B_{1}=20$, and $M=2.5 \times 10^{6}$, PPMPQS is faster than PMPQS (11.9 vs. 16.4).
We conclude that for our implementations PPMPQS can beat PMPQS for numbers of more than 80 (say) decimal digits, but the crossover point strongly depends on the amount of available central memory. For practical reasons (like throughput) it can be profitable to reduce the size of a sieve job on the Cray C90, so even though such a computer has a very large central memory, it is still worthwhile to restrict the size of the upper bound on the primes in the factor base and to have an efficient implementation of a memory-economic method like PPMPQS. This is even more important on workstations, particularly when there are primary and secondary cache memories (as is usual on workstations).

Furthermore, with our PMPQS program we have factored the 99-digit cofactor

\section{6 - 92574904043633682183896384221755952112008347771913}

of the "more wanted" C133 with code 2,914M in the Cunningham table [Wagstaff 1993]. This C133 is the number $\left(2^{457}+2^{229}+1\right) /(5 \times 71293)$; Peter Montgomery had found the 34 -digit prime factor

\section{3}

with ECM, and left the 99-digit composite factor. We decomposed it into the product of the 49- and a 50 -digit primes,

$5845296257595668545524969937697507923682374822769 \times$
28823703291241135239378075616078003806433692452377 
with the help of an eight-processor IBM 9076 SP1, and 69 Silicon Graphics workstations (63 at CWI and 6 at Leiden University). The factor base size was 56976 with $B_{1}=1.5 \times 10^{6}, B_{2} / B_{1}=50$, $M=2 \times 10^{6}$, and $\mathrm{QT}=30$. Parallel processing with good load balancing was effectuated by assigning different polynomials to different workstations. The total amount of sieve time was about 19,500 workstation CPU hours. The physical time for this factorization was about four weeks. This means that we consumed about $40 \%$ of the total CPU capacity of these workstations during that period (assuming that they all are equally fast: in fact, an RS 6000 processor of the IBM SP1 sieved about twice as fast as an SGI workstation). The Gaussian elimination step was carried out on a Cray C90; it required about 0.5 Gbytes of central memory, and one hour CPU time.

As a comparison with a vector computer [te Riele et al. 1991], on a Cray Y-MP we factored a 101digit more wanted Cunningham number with PM$\mathrm{PQS}$ in $475 \mathrm{CPU}$ hours, using $B_{1}=1300000$, with 50179 primes in the factor base, $B_{2} / B_{1}=50, M=$ $4.5 \times 10^{6}$, and QT $=40$ (our PMPQS implementation runs about twice as fast on the Cray C90 as on the Cray Y-MP).

As a comparison with PPMPQS, from the results listed on the right in Table 5 we estimate (based on the assumption that the computing time of PPMPQS approximately doubles if the size of the number increases by three decimal digits) that we would roughly need $10,000 \mathrm{CPU}$ hours of an SGI workstation to factor the 99-digit cofactor of $2,914 \mathrm{M} \mathrm{C133}$, yielding a speed-up factor of about 2 compared to PMPQS. If we would take a factor 1.64 (see the next paragraph) instead of 2, then the time would be less than $4000 \mathrm{CPU}$ hours.

Tables 6 and 7 , on pages 268-271, list the results of our experiments with PPMPQS on eight numbers in the 66-83 digit range on an SGI workstation, and 73 numbers in the 67-88 digit range on a Cray C90 vector computer. Most of these numbers fill gaps in the table found in [Brent and te Riele 1992], and are difficult to factor, having been tried before with ECM without success. The factorizations of some numbers of the form $a^{n} \pm 1$ that are outside the range covered by that reference are also given in Table 7 .

We have varied the parameters $B_{1}, B_{2} / B_{1}$, and $M$ on different numbers (but not in a very systematic way) and kept $\mathrm{QT}=40$ fixed. We observe that the average CPU time for numbers in the $67-88$ digit range varies between 0.4 and 12 CPU hours, so that increasing the number of digits by three gives an increase of the sieve time by a factor of about 1.64. This is smaller than the factor of 2 that is usually observed for PMPQS.

\section{ACKNOWLEDGEMENTS}

We thank Arjen Lenstra, Walter Lioen and Rob Tijdeman for reading the paper and for suggesting several improvements. Walter Lioen helped us with the implementation of our programs on SGI workstations and on Cray vector computers. We gratefully acknowledge the Dutch National Computing Facilities Foundation NCF for the provision of computer time on Cray Y-MP and Cray C90 vector processors. Finally, we acknowledge the help of IBM and the Academic Computing Center Amsterdam (SARA) for providing access to and CPU time on the IBM SP1 at SARA.

\section{REFERENCES}

[Alford and Pomerance 1995] W. R. Alford and C. Pomerance, "Implementing the self-initializing quadratic sieve on a distributed network", pp. 163174 in Number-theoretic and algebraic methods in computer science (NTAMCS '93: Moscow, 1993), edited by A. J. van der Poorten et al., World Scientific, River Edge, NJ, 1995.

[Atkins et al. 1995] D. Atkins, M. Graff, A. K. Lenstra, and P. C. Leyland, "The magic words are squeamish ossifrage", pp. 263-277 in Advances in cryptology (ASIACRYPT '94: Wollongong, 1994), edited by J. Pieprzyk and R. Safavi-Naini, Lecture Notes in Comp. Sci. 917, Springer, Berlin, 1995. 


\begin{tabular}{|c|c|c|c|}
\hline$\#$ & & $n$ & prime factor(s) \\
\hline 1 & C66 from & $77^{53}+1=\mathrm{P} 31 \cdot \mathrm{P} 35$ & $\mathrm{P} 31=8508101816450689975658227843439$ \\
\hline 2 & C67 from & $58^{88}+1=\mathrm{P} 26 \cdot \mathrm{P} 41$ & $\mathrm{P} 26=62057338333442627487392257$ \\
\hline 3 & C67 from & $62^{89}-1=\mathrm{P} 31 \cdot \mathrm{P} 37$ & $\mathrm{P} 31=3916898265747514256035560079891$ \\
\hline 4 & C75 from & $70^{87}+1=\mathrm{P} 29 \cdot \mathrm{P} 46$ & $\mathrm{P} 29=56476537654063551106920429541$ \\
\hline 5 & C79 from & $72^{118}+1=\mathrm{P} 38 \cdot \mathrm{P} 42$ & P38 $=16059490907009321225480347480687832441$ \\
\hline 6 & C82 from & $84^{71}+1=\mathrm{P} 33 \cdot \mathrm{P} 50$ & $\mathrm{P} 33=133184106044570646620234096956423$ \\
\hline 7 & C82 from & $80^{99}+1=\mathrm{P} 32 \cdot \mathrm{P} 51$ & $\mathrm{P} 32=11935171798229644025656192643827$ \\
\hline 8 & C83 from & $92^{87}+1=\mathrm{P} 23 \cdot \mathrm{P} 61$ & $\mathrm{P} 23=10127992394070979564027$ \\
\hline
\end{tabular}

TABLE 6. Parameter choices, timings, and factors for numbers ranging from 66 to 83 decimal digits, factored with PPMPQS on a SGI workstation. Key: $n=$ number to be factored (" $\mathrm{C} x$ from $y$ " means a composite factor of $y$ having $x$ decimal digits); $d=\log _{10} n ; B_{1}=$ upper bound for the primes in the factor base; $B_{2}^{2}=$ upper bound for the input $R$ to SQUFOF (yielding a pp-relation); $n_{f}=$ number of primes in the factor base;

\begin{tabular}{|c|c|c|}
\hline \# & $n$ & prime factor(s) \\
\hline 1 & C67 from $89^{64}+1=\mathrm{P} 24 \cdot \mathrm{P} 44$ & $\mathrm{P} 24=153316525308739316934017$ \\
\hline 2 & $\mathrm{C} 69$ from $50^{122}+1=\mathrm{P} 30 \cdot \mathrm{P} 40$ & $\mathrm{P} 30=276832194921994230575098974137$ \\
\hline 3 & $\mathrm{C} 75$ from $101^{41}+1=\mathrm{P} 32 \cdot \mathrm{P} 43$ & $\mathrm{P} 32=21587227703328821952030527314507$ \\
\hline 4 & $\mathrm{C} 75$ from $110^{41}+1=\mathrm{P} 16 \cdot \mathrm{P} 25 \cdot \mathrm{P} 35$ & $\mathrm{P} 16=3850561614882023 \quad \mathrm{P} 25=7797598239853074057655219$ \\
\hline 5 & C75 from $110^{47}+1=\mathrm{P} 24 \cdot \mathrm{P} 51$ & $\mathrm{P} 24=728424414211828929294823$ \\
\hline 6 & $\mathrm{C} 75$ from $35^{147}+1=\mathrm{P} 35 \cdot \mathrm{P} 40$ & $\mathrm{P} 35=86052439411099140168070862933143801$ \\
\hline 7 & $\mathrm{C} 75$ from $53^{59}-1=\mathrm{P} 24 \cdot \mathrm{P} 51$ & $\mathrm{P} 24=943970114867362247759443$ \\
\hline 8 & C78 from $19^{165}+1=\mathrm{P} 28 \cdot \mathrm{P} 50$ & $\mathrm{P} 28=2481953419044452308291386601$ \\
\hline 9 & C78 from $51^{102}+1=\mathrm{P} 30 \cdot \mathrm{P} 48$ & $\mathrm{P} 30=459028910227193494771112394289$ \\
\hline 10 & $\mathrm{C} 80$ from $86^{58}+1=\mathrm{P} 33 \cdot \mathrm{P} 47$ & $\mathrm{P} 33=129094951090723152084884804969621$ \\
\hline 11 & $\mathrm{C} 80$ from $75^{64}+1=\mathrm{P} 32 \cdot \mathrm{P} 48$ & $\mathrm{P} 32=68799038786512319388821350925569$ \\
\hline 12 & $\mathrm{C} 80$ from $59^{85}-1=\mathrm{P} 36 \cdot \mathrm{P} 44$ & $\mathrm{P} 36=192052183634195717382812875959337681$ \\
\hline 13 & C80 from $76^{123}+1=\mathrm{P} 28 \cdot \mathrm{P} 53$ & $\mathrm{P} 28=1602475801546350975094860307$ \\
\hline 14 & $\mathrm{C} 80$ from $84^{87}-1=\mathrm{P} 40 \cdot \mathrm{P} 41$ & $\mathrm{P} 40=2904043752413366850400636076474517615769$ \\
\hline 15 & $\mathrm{C} 81$ from $18^{103}-1=\mathrm{P} 35 \cdot \mathrm{P} 47$ & $\mathrm{P} 35=15936754604932361311519937275763087$ \\
\hline 16 & $\mathrm{C} 83$ from $82^{68}+1=\mathrm{P} 40 \cdot \mathrm{P} 43$ & $\mathrm{P} 40=9241855378580566956862595601843404638609$ \\
\hline 17 & C83 from $93^{71}+1=$ P34 $\cdot$ P50 & P34 = 1871598891695207952802939248474557 \\
\hline 18 & C84 from $89^{67}-1=\mathrm{P} 41 \cdot \mathrm{P} 44$ & $\mathrm{P} 41=17345460386856072657168883886351357651503$ \\
\hline 19 & C84 from $74^{91}-1=$ P $31 \cdot$ P 54 & $\mathrm{P} 31=6300454649733691099786120178647$ \\
\hline 20 & $\mathrm{C} 85$ from $69^{117}+1=\mathrm{P} 42 \cdot \mathrm{P} 43$ & $\mathrm{P} 42=553775456930001686459646662784000439421893$ \\
\hline 21 & C85 from $98^{91}+1=\mathrm{P} 39 \cdot \mathrm{P} 47$ & P39 $=150856027763097994901861400756223948651$ \\
\hline 22 & $\mathrm{C} 85$ from $80^{58}+1=\mathrm{P} 42 \cdot \mathrm{P} 44$ & $\mathrm{P} 42=587407531780545617292693056474932755332969$ \\
\hline 23 & $\mathrm{C} 85$ from $56^{64}+1=\mathrm{P} 43 \cdot \mathrm{P} 43$ & $\mathrm{P} 43=1120971223480359091305712645673434758493441$ \\
\hline 24 & $\mathrm{C} 85$ from $39^{111}-1=\mathrm{P} 32 \cdot \mathrm{P} 54$ & $\mathrm{P} 32=38661901037861787717347412050407$ \\
\hline 25 & C85 from $77^{95}-1=$ P34.P52 & P34 $=1254200040785197567017611121581711$ \\
\hline 26 & $\mathrm{C} 86$ from $18^{111}+1=\mathrm{P} 35 \cdot \mathrm{P} 51$ & $\mathrm{P} 35=57095169829153516132919139336069139$ \\
\hline 27 & $\mathrm{C} 86$ from $76^{59}+1=\mathrm{P} 39 \cdot \mathrm{P} 47$ & $\mathrm{P} 39=471586815074704431240140019672222092489$ \\
\hline 28 & C 86 from $20^{97}+1=$ P $34 \cdot$ P 52 & $\mathrm{P} 34=2645332912014287669339495089951567$ \\
\hline
\end{tabular}

TABLE 7. Parameter choices, timings, and factorizations for numbers ranging from 67 to 88 decimal digits, 


\begin{tabular}{rccrlllllllrl}
\hline$\#$ & $d$ & $B_{1} / 10^{5}$ & $n_{f}$ & $B_{2} / B_{1}$ & $M / 10^{5}$ & $n_{c}$ & $n_{1}$ & $n_{c, 1}$ & $n_{2}$ & $n_{c, 2}$ & $T_{s}$ \\
\hline 1 & 65.56 & 0.8 & 3911 & 11.25 & 2 & 1493 & 9753 & 1715 & 4102 & 710 & $5.8 \mathrm{~h}$ \\
2 & 66.17 & 0.8 & 3908 & 10 & 1.5 & 1452 & 9433 & 1766 & 3697 & 693 & $4.8 \mathrm{~h}$ \\
3 & 66.83 & 0.8 & 3984 & 10 & 2 & 1214 & 9952 & 2139 & 4238 & 637 & $14.2 \mathrm{~h}$ \\
4 & 74.15 & 3 & 13045 & 20 & 6 & 4840 & 37472 & 4790 & 26391 & 3424 & $55.4 \mathrm{~h}$ \\
\hline 5 & 78.76 & 3 & 12898 & 30 & 5 & 4444 & 44583 & 5104 & 29653 & 3355 & 123 & $\mathrm{~h}$ \\
6 & 81.54 & 5 & 20812 & 20 & 5 & 7992 & 63176 & 8471 & 33614 & 4351 & 173 & $\mathrm{~h}$ \\
7 & 81.70 & 4.5 & 18961 & 20 & 4.5 & 6796 & 55435 & 7229 & 38950 & 4942 & 198 & $\mathrm{~h}$ \\
8 & 82.89 & 5 & 20861 & 20 & 8 & 7387 & 62346 & 8229 & 40035 & 5250 & 273 & $\mathrm{~h}$ \\
\hline
\end{tabular}

$[-M, M]=$ sieve interval; $n_{c}=$ number of complete relations found immediately; $n_{1}=$ number of partial relations; $n_{c, 1}=$ number of complete relations coming from partial relations; $n_{2}=$ number of pp-relations; $n_{c, 2}=$ number of complete relations coming from pp-relations; $T_{s}=$ sieve CPU time. The small-prime variation parameter QT is always 40 .

\begin{tabular}{|c|c|c|c|c|c|c|c|c|c|c|c|}
\hline$\#$ & $d$ & $B_{1} / 10^{5}$ & $n_{f}$ & $B_{2} / B_{1}$ & $M / 10^{5}$ & $n_{c}$ & $n_{1}$ & $n_{c, 1}$ & $n_{2}$ & $n_{c, 2}$ & $T_{s}$ \\
\hline 1 & 66.80 & 2 & 8881 & 30 & 25 & 2945 & 27673 & 2762 & 31855 & 3347 & $0.36 \mathrm{~h}$ \\
\hline 2 & 68.74 & 2.5 & 11086 & 20 & 5 & 3988 & 30107 & 3631 & 27746 & 3476 & $0.46 \mathrm{~h}$ \\
\hline 3 & 74.20 & 3.16 & 13623 & 20 & 6.31 & 4921 & 38371 & 4889 & 29855 & 3822 & $1.22 \mathrm{~h}$ \\
\hline 4 & 74.51 & 3.16 & 13625 & 20 & 6.31 & 5503 & 42284 & 5844 & 17604 & 2297 & $1.16 \mathrm{~h}$ \\
\hline 5 & 74.69 & 1 & 4790 & 60 & 5 & 1005 & 17630 & 1320 & 29502 & 2465 & $2.42 \mathrm{~h}$ \\
\hline 6 & 74.83 & 3 & 12892 & 17 & 25 & 4697 & 37137 & 5388 & 19447 & 2820 & $1.20 \mathrm{~h}$ \\
\hline 7 & 74.92 & 2.5 & 11086 & 36 & 25 & 3339 & 35899 & 3335 & 43531 & 4382 & $1.91 \mathrm{~h}$ \\
\hline 8 & 77.37 & 5 & 20972 & 20 & 25 & 7152 & 54706 & 6444 & 60361 & 7393 & $1.84 \mathrm{~h}$ \\
\hline 9 & 77.56 & 5 & 20888 & 30 & 30 & 7518 & 65930 & 6980 & 60042 & 6453 & $1.41 \mathrm{~h}$ \\
\hline 10 & 79.04 & 5 & 20597 & 30 & 30 & 6596 & 61563 & 6201 & 76295 & 7828 & $2.43 \mathrm{~h}$ \\
\hline 11 & 79.17 & 4 & 16927 & 20 & 1 & 5619 & 45717 & 5584 & 48399 & 6279 & $3.29 \mathrm{~h}$ \\
\hline 12 & 79.17 & 5 & 20895 & 20 & 3 & 6457 & 72272 & 11650 & 37114 & 2802 & $2.68 \mathrm{~h}$ \\
\hline 13 & 79.39 & 3 & 13001 & 166.7 & 3 & 3739 & 68195 & 4113 & 72708 & 5157 & $2.27 \mathrm{~h}$ \\
\hline 14 & 79.87 & 3 & 13011 & 166.7 & 3 & 3323 & 64308 & 3624 & 91150 & 6084 & $3.41 \mathrm{~h}$ \\
\hline 15 & 80.86 & 5 & 20819 & 20 & 6 & 6925 & 57619 & 7050 & 55281 & 6877 & $3.36 \mathrm{~h}$ \\
\hline 16 & 82.82 & 6 & 24598 & 20 & 2.5 & 8522 & 68723 & 8378 & 59901 & 7713 & $4.82 \mathrm{~h}$ \\
\hline 17 & 82.91 & 7 & 28413 & 20 & 2.5 & 11451 & 87010 & 11694 & 40636 & 5271 & $4.38 \mathrm{~h}$ \\
\hline 18 & 83.66 & 8 & 32104 & 25 & 2.5 & 11419 & 96138 & 10894 & 85260 & 9807 & $5.46 \mathrm{~h}$ \\
\hline 19 & 83.98 & 7 & 27980 & 25.7 & 2.5 & 10594 & 93766 & 11327 & 51233 & 6070 & $6.59 \mathrm{~h}$ \\
\hline 20 & 84.10 & 5 & 20713 & 20 & 2.5 & 6175 & 51592 & 5808 & 76377 & 8732 & $5.6 \mathrm{~h}$ \\
\hline 21 & 84.35 & 5 & 20741 & 20 & 2.5 & 6865 & 60444 & 7638 & 72201 & 6256 & $9.8 \mathrm{~h}$ \\
\hline 22 & 84.80 & 5 & 20744 & 20 & 2.5 & 7809 & 57576 & 7457 & 44022 & 5481 & $9.8 \mathrm{~h}$ \\
\hline 23 & 84.87 & 5 & 20790 & 20 & 2.5 & 7153 & 61546 & 7923 & 43044 & 5721 & $8.4 \mathrm{~h}$ \\
\hline & & 5 & 20790 & 40 & 2.5 & 6412 & 73385 & 6960 & 75133 & 7427 & $8.4 \mathrm{~h}$ \\
\hline 24 & 84.92 & 5 & 20930 & 20 & 2.5 & 6434 & 52315 & 5865 & 75217 & 8614 & $6.0 \mathrm{~h}$ \\
\hline 25 & 84.99 & 5 & 20749 & 20 & 2.5 & 7106 & 58607 & 7259 & 53507 & 6389 & $6.8 \mathrm{~h}$ \\
\hline 26 & 85.02 & 5 & 20675 & 20 & 2.5 & 6982 & 61080 & 7920 & 64746 & 5774 & $9.8 \mathrm{~h}$ \\
\hline 27 & 85.02 & 5 & 20792 & 20 & 2.5 & 6679 & 58782 & 7268 & 81258 & 6853 & 11. $\mathrm{h}$ \\
\hline 28 & 85.05 & 5 & 20887 & 20 & 2.5 & 7754 & 65228 & 8990 & 46265 & 4178 & $8.4 \mathrm{~h}$ \\
\hline
\end{tabular}

factored with PPMPQS on a Cray C90 vector computer. Key as in Table 6. Continued overleaf. 


\begin{tabular}{|c|c|c|}
\hline \# & $n$ & prime factor(s) \\
\hline 29 & C86 from $93^{99}-1=\mathrm{P} 31 \cdot \mathrm{P} 55$ & $\mathrm{P} 31=3466732593888008254791613360081$ \\
\hline 30 & C 86 from $58^{93}-1=$ P $32 \cdot$ P 54 & $\mathrm{P} 32=75701865042739143157590250368211$ \\
\hline 31 & C 86 from $56^{96}+1=\mathrm{P} 39 \cdot \mathrm{P} 47$ & $\mathrm{P} 39=232559086557407467762901333407938321409$ \\
\hline 32 & C86 from $92^{84}+1=\mathrm{P} 43 \cdot \mathrm{P} 43$ & $\mathrm{P} 43=2465152715658748428830880994824343639019833$ \\
\hline 33 & C86 from $67^{99}-1=\mathrm{P} 34 \cdot \mathrm{P} 52$ & P34 $=2515208214206285121254951932641469$ \\
\hline 34 & $\mathrm{C} 86$ from $13^{138}+1=\mathrm{P} 29 \cdot \mathrm{P} 57$ & $\mathrm{P} 29=54836637716450236990971812089$ \\
\hline 35 & C 86 from $59^{89}-1=$ P31 $\cdot$ P55 & $\mathrm{P} 31=2689941424488348023848649808389$ \\
\hline 36 & $\mathrm{C} 86$ from $21^{123}+1=\mathrm{P} 39 \cdot \mathrm{P} 47$ & $\mathrm{P} 39=380770063539669474313312691529545132713$ \\
\hline 37 & C86 from $38^{81}-1=$ P36 $\cdot$ P50 & $\mathrm{P} 36=511662075163970762060417538436484323$ \\
\hline 38 & $\mathrm{C} 86$ from $31^{117}-1=\mathrm{P} 39 \cdot \mathrm{P} 47$ & $\mathrm{P} 39=250630033376957433234617073114910871767$ \\
\hline 39 & $\mathrm{C} 86$ from $50^{96}+1=\mathrm{P} 35 \cdot \mathrm{P} 51$ & $\mathrm{P} 35=36774112300765382067961168652800897$ \\
\hline 40 & C 86 from $96^{95}+1=\mathrm{P} 28 \cdot \mathrm{P} 58$ & $\mathrm{P} 28=2418476990688796014581890831$ \\
\hline 41 & C86 from $24^{130}+1=\mathrm{P} 36 \cdot \mathrm{P} 50$ & $\mathrm{P} 36=684989928644194001785075922656446841$ \\
\hline 42 & $\mathrm{C} 86$ from $93^{53}+1=\mathrm{P} 38 \cdot \mathrm{P} 49$ & $\mathrm{P} 38=19192699869550253389095978550167828173$ \\
\hline 43 & C86 from $98^{59}+1=$ P $32 \cdot \mathrm{P} 55$ & $\mathrm{P} 32=29037047448209810589475647292291$ \\
\hline 44 & C 86 from $80^{65}+1=\mathrm{P} 31 \cdot \mathrm{P} 55$ & $\mathrm{P} 31=3416871674919158699528742801241$ \\
\hline 45 & $\mathrm{C} 86$ from $8^{2^{7}}+7^{2^{7}}=\mathrm{P} 42 \cdot \mathrm{P} 44$ & $\mathrm{P} 42=519975935060346660783986052760977025136897$ \\
\hline 46 & C86 from $23^{83}-1=\mathrm{P} 38 \cdot \mathrm{P} 49$ & $\begin{array}{l}\mathrm{P} 44=65757674240355835167624181741955409969833473 \\
\mathrm{P} 38=27736074503263071062950778805992164759\end{array}$ \\
\hline 47 & C 86 from $76^{56}+1=\mathrm{P} 40 \cdot \mathrm{P} 47$ & $\mathrm{P} 40=4868699568817220592890920460964327586529$ \\
\hline 48 & C86 from $47^{67}-1=\mathrm{P} 32 \cdot \mathrm{P} 55$ & $\mathrm{P} 32=21270964162538089013014983761851$ \\
\hline 49 & C86 from $67^{76}+1=\mathrm{P} 42 \cdot \mathrm{P} 45$ & $\mathrm{P} 42=315618216027848486834301078445774290254513$ \\
\hline 50 & C 86 from $39^{81}+1=$ P37.P50 & $\mathrm{P} 37=2443003616566663069989278441133518059$ \\
\hline 51 & C 86 from $22^{95}-1=\mathrm{P} 34 \cdot \mathrm{P} 52$ & $\mathrm{P} 34=9624357919068403555091512367414261$ \\
\hline 52 & C 86 from $76^{117}-1=\mathrm{P} 42 \cdot \mathrm{P} 45$ & $\mathrm{P} 42=606202897105850025527074421945484005533987$ \\
\hline 53 & C86 from $95^{80}+1=\mathrm{P} 38 \cdot \mathrm{P} 49$ & P38 $=45089758099791867831637486244759667041$ \\
\hline 54 & $\mathrm{C} 87$ from $62^{65}+1=\mathrm{P} 34 \cdot \mathrm{P} 53$ & $\mathrm{P} 34=1439106922902522842484110155444391$ \\
\hline 55 & C 87 from $72^{99}+1=\mathrm{P} 28 \cdot \mathrm{P} 59$ & $\mathrm{P} 28=8097540789168990910686588841$ \\
\hline 56 & $\mathrm{C} 87$ from $92^{85}-1=\mathrm{P} 32 \cdot \mathrm{P} 56$ & $\mathrm{P} 32=14285278844357974752432939513571$ \\
\hline 57 & C87 from $30^{95}+1=\mathrm{P} 35 \cdot \mathrm{P} 52$ & $\mathrm{P} 35=80451911996934444483653727156040931$ \\
\hline 58 & C 87 from $50^{100}+1=\mathrm{P} 41 \cdot \mathrm{P} 46$ & $\mathrm{P} 41=58951478878513071930500886762077392077601$ \\
\hline 59 & $\mathrm{C} 87$ from $66^{96}+1=\mathrm{P} 42 \cdot \mathrm{P} 46$ & $\mathrm{P} 42=153055732248039041786999207837459270270017$ \\
\hline 60 & $\mathrm{C} 87$ from $19^{101}-1=\mathrm{P} 25 \cdot \mathrm{P} 62$ & $\mathrm{P} 25=5245647644316863182854571$ \\
\hline 61 & C87 from $33^{85}+1=\mathrm{P} 33 \cdot \mathrm{P} 54$ & $\mathrm{P} 33=249536921989169261065035112257901$ \\
\hline 62 & $\mathrm{C} 87$ from $63^{65}+1=\mathrm{P} 42 \cdot \mathrm{P} 46$ & $\mathrm{P} 42=108410889974425685059575647391841055155451$ \\
\hline 63 & C87 from $42^{99}-1=\mathrm{P} 33 \cdot \mathrm{P} 55$ & $\mathrm{P} 33=234373090934137193434426100841739$ \\
\hline 64 & $\mathrm{C} 87$ from $77^{67}-1=\mathrm{P} 41 \cdot \mathrm{P} 46$ & $\mathrm{P} 41=75024943244844149373705126243013155715853$ \\
\hline 65 & C87 from $84^{59}-1=\mathrm{P} 35 \cdot \mathrm{P} 53$ & $\mathrm{P} 35=11779548019122302808328920808327631$ \\
\hline 66 & $\mathrm{C} 87$ from $26^{129}+1=\mathrm{P} 31 \cdot \mathrm{P} 57$ & $\mathrm{P} 31=3076814278757622588317626405309$ \\
\hline 67 & $\mathrm{C} 87$ from $33^{111}-1=\mathrm{P} 38 \cdot \mathrm{P} 50$ & P38 $=21457939605898871224437297672972660829$ \\
\hline 68 & $\mathrm{C} 87$ from $86^{84}+1=\mathrm{P} 40 \cdot \mathrm{P} 48$ & $\mathrm{P} 40=1039512269081394539159468072656199331337$ \\
\hline 79 & C87 from $85^{65}+1=\mathrm{P} 40 \cdot \mathrm{P} 48$ & $\mathrm{P} 40=4645176624103101144238593467706089788481$ \\
\hline 70 & C87 from $45^{85}+1=\mathrm{P} 36 \cdot \mathrm{P} 52$ & $\mathrm{P} 36=218136090485068920975060625740020221$ \\
\hline 71 & C87 from $87^{93}+1=\mathrm{P} 35 \cdot \mathrm{P} 53$ & $\mathrm{P} 35=65234702723152738657728499902597613$ \\
\hline 72 & C87 from $45^{71}+1=\mathrm{P} 27 \cdot \mathrm{P} 61$ & $\mathrm{P} 27=692298161874034730813881603$ \\
\hline 73 & $\mathrm{C} 88$ from $19^{168}+1=\mathrm{P} 42 \cdot \mathrm{P} 47$ & $\mathrm{P} 42=261688712348581672325146786097393313497473$ \\
\hline
\end{tabular}




\begin{tabular}{|c|c|c|c|c|c|c|c|c|c|c|c|}
\hline$\#$ & $d$ & $B_{1} / 10^{5}$ & $n_{f}$ & $B_{2} / B_{1}$ & $M / 10^{5}$ & $n_{c}$ & $n_{1}$ & $n_{c, 1}$ & $n_{2}$ & $n_{c, 2}$ & $T_{s}$ \\
\hline 29 & 85.11 & 5 & 20810 & 20 & 2.5 & 4923 & 43182 & 4064 & 280566 & 11857 & $8.4 \mathrm{~h}$ \\
\hline 30 & 85.11 & 5 & 20841 & 20 & 2.5 & 5615 & 50651 & 5434 & 182705 & 9822 & $10.7 \mathrm{~h}$ \\
\hline 31 & 85.12 & 6 & 24641 & 20 & 2.5 & 8518 & 67320 & 9253 & 73153 & 6953 & $10.0 \mathrm{~h}$ \\
\hline 32 & 85.12 & 5 & 20651 & 20 & 1.5 & 7269 & 64239 & 8799 & 48843 & 4625 & $9.5 \mathrm{~h}$ \\
\hline 33 & 85.14 & 5 & 20812 & 20 & 1.5 & 5064 & 43981 & 4223 & 263194 & 11614 & $10.7 \mathrm{~h}$ \\
\hline 34 & 85.21 & 5 & 20709 & 20 & 1.5 & 6722 & 56788 & 6924 & 92891 & 7136 & $8.27 \mathrm{~h}$ \\
\hline 35 & 85.26 & 5 & 20859 & 20 & 1.5 & 5101 & 44412 & 4378 & 256996 & 11412 & $11.0 \mathrm{~h}$ \\
\hline 36 & 85.26 & 5 & 20768 & 20 & 1.5 & 7186 & 63721 & 8449 & 57739 & 5154 & $12.4 \mathrm{~h}$ \\
\hline 37 & 85.31 & 5 & 20812 & 20 & 1.5 & 5297 & 45852 & 4584 & 185169 & 10967 & $7.45 \mathrm{~h}$ \\
\hline 38 & 85.31 & 5 & 20576 & 20 & 1.5 & 6107 & 55044 & 6362 & 130553 & 8115 & $13.1 \mathrm{~h}$ \\
\hline 39 & 85.33 & 5 & 20709 & 20 & 1.5 & 6859 & 60552 & 7686 & 68840 & 6177 & $11.5 \mathrm{~h}$ \\
\hline 40 & 85.35 & 5 & 20923 & 20 & 1.5 & 6480 & 55476 & 6546 & 127090 & 7903 & $10.7 \mathrm{~h}$ \\
\hline 41 & 85.37 & 5 & 20672 & 20 & 1.5 & 7221 & 62980 & 8435 & 54345 & 5029 & $9.65 \mathrm{~h}$ \\
\hline 42 & 85.42 & 5 & 20672 & 20 & 1.5 & 5695 & 50790 & 5707 & 139604 & 9308 & $10.4 \mathrm{~h}$ \\
\hline 43 & 85.49 & 5 & 20772 & 20 & 1.5 & 7530 & 63927 & 8600 & 51034 & 4656 & $11.9 \mathrm{~h}$ \\
\hline 44 & 85.52 & 5 & 20634 & 20 & 1.5 & 5556 & 50383 & 5456 & 185347 & 9653 & $13.7 \mathrm{~h}$ \\
\hline 45 & 85.53 & 5 & 20711 & 20 & 2.5 & 5054 & 43759 & 4078 & 270308 & 11587 & $11.4 \mathrm{~h}$ \\
\hline 46 & 85.59 & 5 & 20797 & 20 & 1.5 & 7244 & 63668 & 8524 & 61191 & 5044 & $12.6 \mathrm{~h}$ \\
\hline 47 & 85.70 & 5 & 20712 & 20 & 1.5 & 6637 & 56910 & 6862 & 96694 & 7226 & $10.3 \mathrm{~h}$ \\
\hline 48 & 85.72 & 5 & 20911 & 20 & 1.5 & 6311 & 56349 & 6710 & 120384 & 7895 & $15.4 \mathrm{~h}$ \\
\hline 49 & 85.73 & 6 & 26392 & 2 & 3 & 16159 & 24514 & 9487 & 3153 & 749 & $13.8 \mathrm{~h}$ \\
\hline 50 & 85.92 & 6 & 26363 & 2 & 3 & 16376 & 24473 & 9358 & 7417 & 631 & $12.1 \mathrm{~h}$ \\
\hline 51 & 85.93 & 3 & 13041 & 20 & 1.5 & 4117 & 39602 & 5212 & 39395 & 3713 & $17.4 \mathrm{~h}$ \\
\hline 52 & 85.95 & 3 & 13011 & 20 & 2.5 & 4255 & 40517 & 5390 & 24478 & 3366 & $20.6 \mathrm{~h}$ \\
\hline 53 & 85.98 & 5 & 20756 & 2.4 & 2.5 & 10516 & 22044 & 7450 & 6610 & 2795 & $15.7 \mathrm{~h}$ \\
\hline 54 & 86.04 & 5 & 20840 & 20 & 2.5 & 7153 & 62231 & 8139 & 63273 & 5557 & $14.0 \mathrm{~h}$ \\
\hline 55 & 86.13 & 5 & 20838 & 20 & 2.5 & 7009 & 63089 & 8220 & 57513 & 5620 & $11.9 \mathrm{~h}$ \\
\hline 56 & 86.16 & 5 & 20787 & 22 & 2.5 & 7367 & 63987 & 8559 & 54708 & 4900 & $10.8 \mathrm{~h}$ \\
\hline 57 & 86.18 & 5 & 20688 & 20 & 2.5 & 7447 & 64778 & 8836 & 47154 & 4419 & $10.7 \mathrm{~h}$ \\
\hline 58 & 86.22 & 5 & 20852 & 20 & 2.5 & 6202 & 54180 & 6282 & 144069 & 8376 & $11.6 \mathrm{~h}$ \\
\hline 59 & 86.22 & 5 & 20947 & 20 & 2.5 & 7522 & 63620 & 8412 & 52191 & 5091 & $9.35 \mathrm{~h}$ \\
\hline 60 & 86.27 & 5 & 20978 & 40 & 2.5 & 6773 & 79489 & 8184 & 75416 & 6035 & $14.2 \mathrm{~h}$ \\
\hline 61 & 86.29 & 5 & 20797 & 40 & 2.5 & 6387 & 72868 & 6909 & 116000 & 7520 & $11.1 \mathrm{~h}$ \\
\hline 62 & 86.38 & 5 & 20754 & 40 & 2.5 & 6881 & 76861 & 7638 & 86915 & 6253 & $6.72 \mathrm{~h}$ \\
\hline 63 & 86.43 & 5 & 20920 & 40 & 2.5 & 7177 & 76854 & 7706 & 82085 & 6054 & $8.81 \mathrm{~h}$ \\
\hline 64 & 86.45 & 5 & 20631 & 40 & 2.5 & 6329 & 74485 & 7262 & 92362 & 7046 & $14.6 \mathrm{~h}$ \\
\hline 65 & 86.63 & 5 & 20902 & 80 & 2.5 & 5806 & 85167 & 6249 & 148784 & 8876 & $13.8 \mathrm{~h}$ \\
\hline 66 & 86.64 & 6 & 24404 & 100 & 3 & 7564 & 124510 & 9691 & 89594 & 7355 & $13.2 \mathrm{~h}$ \\
\hline 67 & 86.69 & 6 & 24573 & 100 & 3 & 7803 & 122935 & 9614 & 89375 & 7369 & $14.1 \mathrm{~h}$ \\
\hline 68 & 86.70 & 6 & 24495 & 100 & 3 & 6571 & 105037 & 7010 & 149698 & 11272 & $12.1 \mathrm{~h}$ \\
\hline 69 & 86.73 & 6 & 24538 & 100 & 3 & 7635 & 120888 & 9389 & 99930 & 7811 & $11.5 \mathrm{~h}$ \\
\hline 70 & 86.75 & 6 & 24374 & 100 & 3 & 7827 & 126178 & 9899 & 80444 & 6862 & $14.7 \mathrm{~h}$ \\
\hline 71 & 86.82 & 6 & 24615 & 100 & 3 & 6532 & 121187 & 9864 & 167590 & 8507 & $11.8 \mathrm{~h}$ \\
\hline 72 & 86.96 & 6 & 24658 & 100 & 3 & 7762 & 116023 & 8546 & 126334 & 8798 & $7.66 \mathrm{~h}$ \\
\hline 73 & 87.54 & 5 & 20604 & 100 & 1 & 6101 & 94651 & 6605 & 108893 & 8048 & $12.1 \mathrm{~h}$ \\
\hline
\end{tabular}


[Bollobás 1985] B. Bollobás, Random graphs, Academic Press, London and Orlando, FL, 1985.

[Brent and te Riele 1992] R. P. Brent and H. J. J. te Riele, "Factorizations of $a^{n} \pm 1,13 \leq a<$ 100", Technical Report NM-R9212, Centrum voor Wiskunde en Informatica, Kruislaan 413, 1098 SJ Amsterdam, The Netherlands, June 1992. Updates to this report have appeared as CWI Report NMR9419, September 1994 and CWI Report NMR9609, March 1996, with P. L. Montgomery as a coauthor. The complete tables, incorporating these updates, are available at $\mathrm{ftp}: / /$ nimbus.anu.edu.au/ pub/Brent/factors or ftp://ftp.cwi.nl/pub/herman/ factors.

[Bressoud 1989] D. M. Bressoud, Factorization and primality testing, Undergraduate Texts in Math., Springer, New York, 1989.

[Davis and Holdridge 1983] J. A. Davis and D. B. Holdridge, "Factorization using the quadratic sieve algorithm", Technical Report Sand 83-1346, Sandia National Laboratories, Albuquerque, NM, 1983.

[Denny 1993] T. F. Denny, "Faktorisieren mit dem quadratischen Sieb", Master's thesis, Universität des Saarlandes, Saarbrücken, 1993.

[Denny et al. 1994] T. F. Denny, B. Dodson, A. K. Lenstra, and M. S. Manasse, "On the factorization of RSA-120", pp. 166-174 in Advances in Cryptology (CRYPTO '93: Santa Barbara, CA, 1993), edited by D. R. Stinson, Lecture Notes in Comp. Sci. 773, Springer, Berlin, 1994.

[Erdős and Rényi 1959] P. Erdős and A. Rényi, "On random graphs I", Publ. Math. Debrecen 6 (1959), 290-297.

[Erdős and Rényi 1960] P. Erdős and A. Rényi, "On the evolution of random graphs", Publ. Math. Inst. Hungar. Acad. Sci. 5 (1960), 17-61.

[Erdős and Rényi 1961] P. Erdős and A. Rényi, "On the evolution of random graphs", Bull. Inst. Int. Statist. Tokyo 38 (1961), 343-347.

[Kraitchik 1929] M. Kraitchik, Recherches sur la théorie des nombres, II, Gauthier-Villars, Paris, 1929.

[Lehmer and Powers 1931] D. H. Lehmer and R. E. Powers, "On factoring large numbers", Bull. Amer. Math. Soc. 37 (1931), 770-776.
[Lenstra and Lenstra 1993] A. K. Lenstra and H. W. Lenstra, Jr. (editors), The development of the number field sieve, Lecture Notes in Math. 1554, Springer, Berlin, 1993.

[Lenstra and Manasse 1994] A. K. Lenstra and M. S. Manasse, "Factoring with two large primes", Math. Comp. 63 (1994), 785-798.

[Morrison and Brillhart 1975] M. A. Morrison and J. Brillhart, "A method of factoring and the factorization of $F_{7}$ ", Math. Comp. 29 (1975), 183-205.

[Parkinson and Wunderlich 1984] D. Parkinson and W. Wunderlich, "A compact algorithm for Gaussian elimination over $\mathrm{GF}(2)$ implemented on highly parallel computers", Parallel Comput. 1 (1984), 65-73.

[Paton 1969] K. Paton, "An algorithm for finding a fundamental set of cycles of a graph", Comm. ACM 12 (1969), 514-518.

[Peralta $\geq 1997] \quad$ R. Peralta, "Implementation of the hypercube variation of the multiple polynomial quadratic sieve". To appear.

[Pomerance 1982] C. Pomerance, "Analysis and comparison of some integer factoring algorithms", pp. 89-139 in Computational methods in number theory, I, edited by H. W. Lenstra, Jr. and R. Tijdeman, Mathematisch Centrum, Amsterdam, 1982.

[Pomerance 1985] C. Pomerance, "The quadratic sieve factoring algorithm", pp. 169-182 in Advances in cryptology (EUROCRYPT '84: Paris, 1984), edited by T. Beth et al., Lecture Notes in Comp. Sci. 209, Springer-Verlag, New York, 1985.

[Pomerance et al. 1988] C. Pomerance, J. W. Smith, and R. Tuler, "A pipeline architecture for factoring large integers with the quadratic sieve algorithm", SIAM J. Comput. 17 (1988), 387-403.

[te Riele et al. 1989] H. J. J. te Riele, W. M. Lioen, and D. T. Winter, "Factoring with the quadratic sieve on large vector computers", J. Comp. Appl. Math. 27 (1989), 267-278.

[te Riele et al. 1991] H. te Riele, W. Lioen, and D. Winter, "Factorization beyond the googol with MPQS on a single computer", CWI Quarterly 4 (March 1991), 69-72. 
[Riesel 1985] H. Riesel, Prime numbers and computer methods for factorization, Progress in Math. 126, Birkhäuser, Boston, 1985.
[Silverman 1987] R. D. Silverman, "The multiple polynomial quadratic sieve", Math. Comp. 48 (1987), 329-339.

[Wagstaff 1993] S. S. Wagstaff, Jr., December 13, 1993. Regular letter to subscribers describing the state of the Cunningham factorization project.

Henk Boender, Department of Mathematics and Computer Science, University of Leiden, P.O. Box 9512, 2300 RA Leiden, The Netherlands (henkb@wi.leidenuniv.nl), and Centrum voor Wiskunde en Informatica, P.O. Box 94079, 1090 GB Amsterdam, The Netherlands (henkb@cwi.nl)

Herman J. J. te Riele, Centrum voor Wiskunde en Informatica, P.O. Box 94079, 1090 GB Amsterdam, The Netherlands (herman@cwi.nl)

Received October 10, 1995; accepted September 5, 1996 